1997

\title{
Symmetrically Continuous Function Which Is Not Countably Continuous
}

Krzysztof Ciesielski

West Virginia University, krzysztof.ciesielski@mail.wvu.edu

Follow this and additional works at: https://researchrepository.wvu.edu/faculty_publications

Part of the Mathematics Commons

\section{Digital Commons Citation}

Ciesielski, Krzysztof, "Symmetrically Continuous Function Which Is Not Countably Continuous" (1997). Faculty Scholarship. 824.

https://researchrepository.wvu.edu/faculty_publications/824

This Article is brought to you for free and open access by The Research Repository @ WVU. It has been accepted for inclusion in Faculty Scholarship by an authorized administrator of The Research Repository@WVU. For more information, please contact ian.harmon@mail.wvu.edu. 
Krzysztof Ciesielski and Marcin Szyszkowski, Department of Mathematics, West Virginia University, Morgantown, WV 26506-6310, USA, email: kcies@@wvnvms.wvnet.edu and marcin@@math.wvu.edu

\title{
A SYMMETRICALLY CONTINUOUS FUNCTION WHICH IS NOT COUNTABLY CONTINUOUS
}

\begin{abstract}
We construct a symmetrically continuous function $f: \mathbb{R} \rightarrow \mathbb{R}$ such that for some $X \subset \mathbb{R}$ of cardinality continuum $f \mid X$ is of SierpińskiZygmund type. In particular such an $f$ is not countably continuous. This gives an answer to a question of Lee Larson.
\end{abstract}

\section{Preliminaries}

This paper concerns the relation between the following two notions of generalized continuity. (See [2, Ch 3, 70-84] or [3].)

We say that a function $f: \mathbb{R} \rightarrow \mathbb{R}$ is symmetrically continuous at point $x \in \mathbb{R}$ if

$$
\lim _{k \rightarrow 0} f(x+k)-f(x-k)=0 .
$$

Function $f: \mathbb{R} \rightarrow \mathbb{R}$ is symmetrically continuous if it is symmetrically continuous at every point $x \in \mathbb{R}$.

For $X \subset \mathbb{R}$ a function $f: X \rightarrow \mathbb{R}$ is countably continuous if there is a countable cover $\left\{X_{n}: n \in \mathbb{N}\right\}$ of $X$ (by arbitrary sets) such that each restriction $f \mid X_{n}$ is continuous.

It is known that a symmetrically continuous function $f: \mathbb{R} \rightarrow \mathbb{R}$ is relatively close to being continuous. For example the set of points of discontinuity of $f$ is nowhere dense and of measure zero. (See e.g. [3, Sec. 2.7].) Thus, Lee Larson (private communication) asked whether every symmetrically continuous function is countably continuous. The main aim of this note is to give a negative answer to this question.

We will also use the following notion. For $X \subset \mathbb{R}$ a function $f: X \rightarrow \mathbb{R}$ is said to be of Sierpinski-Zygmund type if $f \mid Y$ is discontinuous for every $Y \subset X$ of cardinality $\mathfrak{c}$, the cardinality of $\mathbb{R}$.

Key Words: symmetric continuity, countable continuity, Sierpiński-Zygmund functions

Mathematical Reviews subject classification: Primary 26A15; Secondary 26A03.

Received by the editors April 26, 1996 
The following fact describes the basic relation between these classes of functions.

Fact 1.1 Let $f: \mathbb{R} \rightarrow \mathbb{R}$. If there exists $X \subset \mathbb{R}$ of cardinality $\mathfrak{c}$ such that $f \mid X$ is of Sierpiński-Zygmund type then $f \mid X$ and $f$ are not countably continuous.

Proof. If $\left\{X_{n} \subset X: n \in \mathbb{N}\right\}$ is a cover of $X$ then there exists $n \in \mathbb{N}$ such that $X_{n}$ has cardinality $\mathfrak{c}$. (Since the cofinality of $\mathfrak{c}$ is uncountable.) Thus, $f \mid X_{n}$ is discontinuous, as $f \mid X$ is of Sierpiński-Zygmund type.

\section{Technical Lemmas}

The construction presented below is, in a big part, based on the technique developed in [1]. In particular, the following lemmas are the modifications of their counterparts from [1].

In what follows we will use the following notation. For $A, B \subset \mathbb{R}$ we define

$$
2 A=\{2 x: x \in A\} \quad \text { and } \quad A+B=\{x+y: x \in A \& y \in B\} .
$$

In the case when $B=\{b\}$, we write $A+b$ instead of $A+\{b\}$. The symbol $\chi_{A}$ will denote the characteristic function of a set $A \subset \mathbb{R}$.

The set of centers of symmetry of a set $A \subset \mathbb{R}$ will be denoted by $A^{\star}$, i.e.,

$$
A^{\star}=\{x \in \mathbb{R}:(\forall k \in \mathbb{R})(x+k \in A \Longleftrightarrow x-k \in A)\} .
$$

For any function $f: X \rightarrow \mathbb{R}$ with $X \subset \mathbb{R}$ the symbol $C(f)$ will stand for the set of continuity points of $f$ and $D(f)$ for the set of points of discontinuity. Thus, $D(f)=\mathbb{R} \backslash C(f)$.

Lemma 2.1 Let $h: \mathbb{R} \rightarrow \mathbb{R}$ be symmetrically continuous and $\left\{A_{\alpha}\right\}_{\alpha \in \mathcal{A}}$ be a family of disjoint subsets of $\mathbb{R}$ such that for every $x \in \mathbb{R}$

$$
\lim _{y \rightarrow x} h(y)=0 \quad \text { or } \quad x \in \bigcap_{\alpha \in \mathcal{A}} A_{\alpha}^{\star}
$$

If $r_{\alpha} \in[0,1]$ for every $\alpha \in \mathcal{A}$ then the function

$$
f=h \cdot \sum_{\alpha \in \mathcal{A}} r_{\alpha} \chi_{A_{\alpha}}
$$

is symmetrically continuous. 
Proof. This is a modification of Lemma 2 from [1]. First note that $0 \leq$ $\sum_{\alpha \in \mathcal{A}} r_{\alpha} \chi_{A_{\alpha}} \leq 1$ since the sets $A_{\alpha}$ are disjoint. Let $x \in \mathbb{R}$.

If $\lim _{y \rightarrow x} h(y)=0$ then

$$
\lim _{y \rightarrow x} f(y)=\lim _{y \rightarrow x}\left(h \cdot \sum_{\alpha \in \mathcal{A}} r_{\alpha} \chi_{A_{\alpha}}\right)(y)=0
$$

since $\sum_{\alpha \in \mathcal{A}} r_{\alpha} \chi_{A_{\alpha}}$ is bounded. Hence $f$ is symmetrically continuous at $x$.

If $x \in \bigcap_{\alpha \in \mathcal{A}} A_{\alpha}^{\star}$ then $\sum_{\alpha \in \mathcal{A}} r_{\alpha} \chi_{A_{\alpha}}(x-k)=\sum_{\alpha \in \mathcal{A}} r_{\alpha} \chi_{A_{\alpha}}(x+k)$ for every $k \in \mathbb{R}$, and so

$$
\lim _{k \rightarrow 0} f(x+k)-f(x-k)=\lim _{k \rightarrow 0}[h(x+k)-h(x-k)] \sum_{\alpha \in \mathcal{A}} r_{\alpha} \chi_{A_{\alpha}}(x+k)=0 .
$$

Thus, once again, $f$ is symmetrically continuous at $x$.

Lemma 2.2 If $G \subset \mathbb{R}$ is an additive subgroup then $G \subset(2 G+x)^{\star}$ for every $x \in G$.

Proof. Let $g \in G, a \in 2 G+x$ and $b$ be a point symmetric to $a$ with respect to $g$, i.e., such that $a+b=2 g$. We have to prove that $b \in 2 G+x$.

So, let $z \in G$ be such that $a=2 z+x$. Then

$$
b=2 g-a=2 g-(2 z+x)=2(g-z-x)+x \in 2 G+x
$$

since $g, x, z \in G$.

Lemma 2.3 If $G \subset \mathbb{R}$ is an additive subgroup then the sets $\{2 G+x: x \in G\}$ form a partition of $G$.

Proof. This is just the usual coset decomposition of $G$ using the subgroup $2 G$. To see it explicitly, assume that for some $x, y \in G$ there exists $z \in$ $(2 G+x) \cap(2 G+y)$. We have to prove that $2 G+x=2 G+y$, i.e., that $x-y \in 2 G$. So, let $a, b \in G$ be such that $z=2 a+x=2 b+y$. Then $x-y=2(b-a) \in 2 G$.

Lemma 2.4 There exists a symmetrically continuous function $h: \mathbb{R} \rightarrow \mathbb{R}$ with the property that

(i) $C(h)=h^{-1}(0)$,

(ii) $D(h)$ is an additive subgroup of $\mathbb{R}$, and

(iii) there exists a subset $X$ of $D(h)$ of cardinality $\mathfrak{c}$ such that

$$
(2 D(h)+x) \cap(2 D(h)+y)=\emptyset \quad \text { for every distinct } x, y \in X .
$$


Proof. Chlebík in [1] constructed a symmetrically continuous function $h: \mathbb{R} \rightarrow \mathbb{R}$ satisfying (i) ${ }^{1}$ and (ii) for which there exists a set $X \subset D(h)$ of cardinality $\mathfrak{c}$ with the property that

$$
2 D(h)+H_{1} \neq 2 D(h)+H_{2} \quad \text { for every distinct } H_{1}, H_{2} \subset X .
$$

In particular, if $x \in X$ and $y \in X$ are distinct and $H_{1}=\{x\}, H_{2}=\{y\}$, then

$$
2 D(h)+x=2 D(h)+H_{1} \neq 2 D(h)+H_{2}=2 D(h)+y .
$$

So, by Lemma $2.3,(2 D(h)+x) \cap(2 D(h)+y)=\emptyset$.

\section{Main result}

Theorem 3.1 There exists a symmetrically continuous function $f: \mathbb{R} \rightarrow \mathbb{R}$ and a subset $X$ of $\mathbb{R}$ of cardinality $\mathfrak{c}$ such that $f \mid X$ is of a Sierpiński-Zygmund type.

Proof. Let

$$
\mathcal{D}=\left\{\langle g, G\rangle: G \text { is a } G_{\delta} \text { subset of } \mathbb{R} \text { and } g: G \rightarrow \mathbb{R} \text { is continuous }\right\}
$$

and let $\left\langle\left\langle G_{\alpha}, g_{\alpha}\right\rangle: \alpha<\mathfrak{c}\right\rangle$ be an enumeration of $\mathcal{D}$. Also, let $h: \mathbb{R} \rightarrow \mathbb{R}$ and $X$ be from Lemma 2.4 and pick a one-to-one enumeration $\left\langle x_{\alpha}: \alpha<\mathfrak{c}\right\rangle$ of $X$.

By transfinite induction on $\alpha<\mathfrak{c}$ define a sequence $\left\langle r_{\alpha}: \alpha<\mathfrak{c}\right\rangle$ such that the following inductive condition is satisfied for every $\alpha<\mathfrak{c}$ :

$$
r_{\alpha} \in[0,1] \backslash\left\{\frac{g_{\beta}\left(x_{\alpha}\right)}{h\left(x_{\alpha}\right)}: \beta \leq \alpha \& x_{\alpha} \in G_{\beta}\right\} .
$$

(Note that $h\left(x_{\alpha}\right) \neq 0$ since $x_{\alpha} \in D(h)=\mathbb{R} \backslash h^{-1}(0)$.)

Now let $A_{\alpha}=2 D(h)+x_{\alpha}$ for every $\alpha<\mathfrak{c}$ and notice that, by Lemma 2.4, the sets $\left\{A_{\alpha}: \alpha<\mathfrak{c}\right\}$ are disjoint. Define

$$
f=h \cdot \sum_{\alpha \in \mathcal{A}} r_{\alpha} \chi_{A_{\alpha}}
$$

Thus, $f$ is a well defined real function. Moreover, by Lemma 2.2,

$$
D(h) \subset \bigcap_{\alpha<\mathfrak{c}}\left(2 D(h)+x_{\alpha}\right)^{\star}=\bigcap_{\alpha<\mathfrak{c}} A_{\alpha}^{\star},
$$

\footnotetext{
${ }^{1}$ Chlebík remarks only that $h$ is continuous at every point of $h^{-1}(0)$, implying that $h^{-1}(0) \subset C(h)$. However $h^{-1}(0)$ is clearly dense in $\mathbb{R}$. So $\mathbb{R} \backslash h^{-1}(0) \subset D(h)$ and indeed $C(h)=h^{-1}(0)$.
} 
since $D(h)$ is an additive subgroup of $\mathbb{R}$. So, by Lemma $2.1, f$ is symmetrically continuous, since $\mathbb{R} \backslash D(h)=C(h)=h^{-1}(0)$, implying (1). It remains to show that is $f \mid X$ is of Sierpiński-Zygmund type.

For this, by way of contradiction, assume that there exists $Y \subset X$ of cardinality $\mathfrak{c}$ such that $f \mid Y$ is continuous. Then there exists a $G_{\delta}$ set $G \subset \mathbb{R}$ containing $Y$ and a continuous function $g: G \rightarrow \mathbb{R}$ such that $g|Y=f| Y$. In particular, $\langle g, G\rangle \in \mathcal{D}$, and there exists $\beta<\mathfrak{c}$ such that $\langle g, G\rangle=\left\langle g_{\beta}, G_{\beta}\right\rangle$. Also, since $Y$ has cardinality $\mathfrak{c}$, there exists $\alpha<\mathfrak{c}, \alpha \geq \beta$, such that $x_{\alpha} \in Y$. But $h\left(x_{\alpha}\right) \neq 0$, since $x_{\alpha} \in X \subset D(h)=\mathbb{R} \backslash C(h)=\mathbb{R} \backslash h^{-1}(0)$. So, by (2), and the fact that $x_{\alpha} \in A_{\alpha}$

$$
f\left(x_{\alpha}\right)=h\left(x_{\alpha}\right) \cdot r_{\alpha} \neq h\left(x_{\alpha}\right) \frac{g_{\beta}\left(x_{\alpha}\right)}{h\left(x_{\alpha}\right)}=g_{\beta}\left(x_{\alpha}\right)=g\left(x_{\alpha}\right)
$$

contradicting $g|Y=f| Y$.

Corollary 3.2 There is a symmetrically continuous function $f: \mathbb{R} \rightarrow \mathbb{R}$ which is not countably continuous.

Proof. By Theorem 3.1 and Fact 1.1.

Notice also that in Fact 1.1 and Corollary 3.2 we can conclude also that $f$ is not $\kappa$-continuous (the graph of $f$ cannot be covered by the graphs of $\kappa$ many continuous functions) where $\kappa$ is less then the cofinality of $\mathfrak{c}$.

It is also worth to mention that neither Chlebík's theorem nor Corollary 3.2 can be generalized for the class of symmetrically differentiable functions, i.e., the functions $f: \mathbb{R} \rightarrow \mathbb{R}$ for which the limit

$$
\lim _{k \rightarrow \infty} \frac{f(x+k)-f(x-k)}{2 k}
$$

exists and is finite for every $x \in \mathbb{R}$. This follows from a theorem of Charzyński [3, Thm 2.9], since the set $D(f)$ for every such function is at most countable. On the other hand, we do not know whether the same is true if we allow the limit (3) to be infinite.

\section{References}

[1] M. Chlebík, There are $2^{\mathfrak{c}}$ symmetrically continuous functions, Proc. Amer. Math. Soc. 113 (1991), 683-688.

[2] B. S. Thomson, Real Functions, Springer-Verlag, Lecture Notes in Math. 1170, 1985.

[3] B. S. Thomson, Symmetric properties of real functions, Marcel Dekker, Inc. 1993. 